\title{
Malignant Restenosis and Progression of Disease in Psoriatic Patients Undergoing Coronary Interventions: Is it the Koebner's Phenomena to Blame?
}

\author{
Vineet Bhatia, MD, DM, DNB, MNAMS'*; Parneesh Arora, MD, DM, DNB, MNAMS'; Vineet Relhan, MD²; \\ Upendra Kaul, MD, DM, FACC, FSCAI, FAMS ${ }^{3}$
}

'Department of Cardiology, Fortis Hospital, Noida, Uttar Pradesh, India

${ }^{2}$ Department of Dermatology, Maulana Azad Medical College, New Delhi, India

${ }^{3}$ Department of Cardiology, Batra Heart Care Centre, New Delhi, India

"Corresponding author

Vineet Bhatia, MD, DM, DNB, MNAMS

Senior Consultant, Department of Cardiology, Fortis Hospital, B-22, Sector 62, Noida, Uttar Pradesh, India; E-mail: vinmonica@yahoo.co.in

\section{Article information}

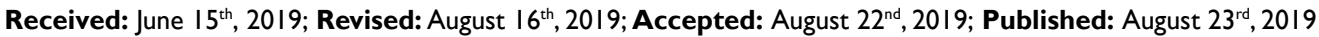

\section{Cite this article}

Bhatia V,Arora P, Relhan V, Kaul U. Malignant restenosis and progression of disease in psoriatic patients undergoing coronary interventions: Is it the koebner's phenomena to blame? Heart Res Open J. 2019;6(I): 15-17. doi: 10.17।40/HROJ-6-150

\section{| ABSTRACT |}

Severe psoriasis is associated with an enhanced risk of cardiovascular (CV) diseases. The potential impact of psoriasis on the prognosis following coronary revascularization (percutaneous coronary intervention (PCI) or surgical) is not well studied and sparse data available in literature points towards a grim long-term prognosis. The following two cases highlight this phenomenon. We also discuss the plausible reasons responsible for the poor prognosis and propose some possible mechanisms for the same.

\section{INTRODUCTION}

$\mathrm{P}$ soriasis is a chronic, immune-mediated inflammatory disorder affecting about $1-3 \%$ of the population worldwide. It is now considered a systemic disease affecting several body systems rather than a disorder characterized by skin and joint manifestations. Not only is the prevalence of established traditional cardiovascular (CV) risk factors higher in patients with severe psoriasis they also exhibit increased rates of myocardial infarction, atrial fibrillation, coronary revascularization, stroke, $\mathrm{CV}$ death, and overall mortality, suggesting a potential role of shared pathways in both psoriasis and atherosclerosis. ${ }^{1}$ However, whether psoriasis also poses as a significant risk factor for repeated coronary interventions remains, largely unexplored. Only one study ${ }^{2}$ has looked at the impact of psoriasis on post-percutaneous coronary intervention (PCI) prognosis and results have raised several valid concerns that this disease may pose in patients undergoing PCI.

We came across two patients who underwent repeated coronary interventions (surgical/percutaneous) due to recurrent restenosis. One thing common between the two was the presence of severe psoriasis. The following discussion looks at the patient profiles and the plausible mechanisms and role of psoriasis in perpetuating this malignant restenosis.

\section{CASE REPORT |}

Case I

A 68-years-old lady normotensive, non-diabetic and a known case of severe psoriasis presented with unstable angina. She had suffered an anteroseptal myocardial infarction (MI) a year ago. She underwent coronary angiography which revealed 100\% ostial left anterior descending artery (LAD) occlusion and significant left main coronary artery (LMCA) and proximal left circumflex disease $(\mathrm{LCx})$. Following a stress thallium study which revealed no significant reversible ischemia in the LAD territory, she underwent percutaneous transluminal coronary angioplasty (PTCA) and stenting from LMCA to LCX using a 4X $23 \mathrm{~mm}$ vision stent (Abbot Vascular, Santa Clara, CA, USA). She was also on methotrexate and phototherapy for her psoriatic skin lesions. The patient had clinically documented Koebner's phenomena. She presented 3-months later with recurrence of angina and a check angiogram 
revealed $90 \%$ in-stent restenosis (ISR) in the proximal part of the LMCA stent. This time she underwent repeat angioplasty and stenting from LMCA to LCX using a 4X $28 \mathrm{~mm}$ Xience V stent (Abbot Vascular, Santa Clara, CA, USA). Eight months later she once again had recurrence of angina and an angiogram revealed 90\% LMCA in-stent restenosis following which PTCA with drugeluting balloon (SeQuent Please, B. Braun, Berlin, Germany) to LMCA was done. One month later the patient had sudden cardiac death at home.

\section{Case 2}

A 76-years-old gentleman known diabetic, hypertensive with psoriasis and psoriatic arthropathy presented with angina in June 2001. Coronary angiography (CAG) was performed revealing double vessel disease following which he underwent PTCA and stent to LAD using a Tetra 3X13 mm bare metal stent (Guidant Corporation, Santa Clara, CA, USA) and balloon angioplasty to posterior descending artery (PDA). He had recurrence of angina in September 2001 and then underwent coronary artery bypass grafting (CABG) (Left Internal Mammary Artery-LIMA to LAD, Saphenous venous graft-respiratory syncytial virus glycoprotein (RSVG) to diagonal 1 and radial artery to right coronary arteryRCA). Four months later in view of angina a repeat angiogram was carried out which revealed occluded left internal mammary artery (LIMA) and radial grafts, and underwent redo coronary artery bypass grafting (CABG) using venous grafts. The fact that the sites of occlusion of these grafts were the distal anastomotic sites the possibility of this being a surgical technical complication cannot be negated totally with certainty. In August 2004, CAG done, for recurrence of angina revealed blocked SVG grafts, normal LMCA, LAD 80\% instent restenosis, D1 90\% ostial disease, LCx 50\% proximal lesion and PDA $100 \%$ occlusion. He subsequently was on medical management with partial relief. Following an angiogram in January 2006 he underwent PTCA and stent to LAD using Cypher select 3.5X28 mm stent and plain balloon angioplasty to D1. This was then followed by plain balloon angioplasty to LAD in June 2008 as LAD revealed $100 \%$ ISR (the procedure performed as patient had rest angina). At this time diffuse disease in LAD was noted distal to the stent and hence a check shoot one month later was planned, which revealed 100\% LAD occlusion. He subsequently was put on optimal medical therapy however succumbed following stroke related complications in 2016.

\section{DISCUSSION}

The normal healing response of the arterial injury following coronary stenting comprises of two main processes, the formation of new intima and reendothelialization of the injured vessel surface. $^{3,4}$ The earliest reaction following deployment of baremetal stent is vessel injury which results in formation of plateletrich thrombus. At the same time acute inflammatory cell infiltrate occurs within the thrombus and the adjoining vessel wall. This is followed by macrophage infiltration which induces cytokine and growth factor release. The growth factors lead to smooth muscle proliferation and migration. These processes in the animal models occur within 14 to 21-days. Simultaneously, matrix deposition occupies approximately $50 \%$ of the neo-intimal growth by 28 days in animals, whereas in man this process takes 6-9-months. Thereafter the neo-intima begins to regress as it gets replaced by type 1 collagen. ${ }^{3,4}$ Finally there is endothelialization of the injured vessel. At this stage endothelial cells repopulate the luminal surface thus forming a protective barrier against thrombus formation.

When this healing response gets exaggerated it leads to restenosis which is a major limitation with coronary stenting requiring patients to undergo repeated revascularization procedures or even surgery. It is proposed that restenosis is a multi-factorial process caused by a number of overlapping processes, such as neo-intimal hyperplasia due to increased inflammation, increased smooth muscle cell proliferation, and/or increased extracellular matrix deposition. ${ }^{3,4}$

What is unique to Psoriasis? In a Danish study, ${ }^{2}$ which looked at the prognosis after PCI in psoriatic patients found that there was a non-significant trend $(p=0.23)$ for increased repeat revascularization rates in patients with psoriasis compared to patients without psoriasis. The incidence rates (IRs) were 66.9 (CI 65.6-68.3), 71.3 (CI 62.2-81.6), and 75.0 (CI 57.7-97.5), for patients without psoriasis, with mild psoriasis, and with severe psoriasis, respectively. The corresponding adjusted HRs were 1.05 (CI $0.89-1.22$ ) and 1.14 (CI 0.85-1.52) for mild and severe psoriasis. Inflammation has also been suggested to contribute to postPCI complications including restenosis, in-stent thrombosis and mortality. Very limited data are available, however, on the impact of inflammatory diseases on the prognosis after PCI. A small study of patients with systemic lupus erythematosus demonstrated a worse 1-year outcome compared to controls while a very small study of patients with rheumatoid arthritis found no differences between groups. ${ }^{4,5}$

Apart from being an immune-mediated disease characterized by a heightened inflammatory state which could lead to enhanced and exaggerated inflammatory reaction to vessel injury psoriatic patient's exhibits unique phenomena called Koebner's phenomenon. This is characterized by development of new skin lesions wherever trauma occurs in uninvolved areas. Trauma caused by friction and lacerations are known to induce this phenomena. ${ }^{6}$ The Koebner's response is more likely to occur when psoriasis is unstable or flaring and less likely with quiescent or resolving psoriasis. More severe injury may result in more extensive lesions. Histopathology of such lesions also reveals an exaggerated immune response. ${ }^{6}$ We strongly feel that in the presence of an already heightened inflammatory condition the vessel trauma caused by the intervention induces a Koebner's like response in the vessel wall (due to trauma at site of intervention) and leads to an exaggerated inflammatory state with subsequent higher rates of restenosis. However, this phenomena needs to be proven histo-pathologically and at the present time is a postulation. 
It also highlights the need for a proper history taking by the cardiac sciences team so that such subsets can be identified and counseled accordingly.

\section{CONCLUSION}

Autoimmune inflammatory disorders as psoriasis are associated with increased risk of adverse cardiovascular events. Interventional cardiac therapies in such patients might also be fraught with higher failure rates in the long-term due to enhanced rate of restenosis secondary to a hyper-intense inflammatory reaction. Although not proven, Koebner's phenomena like response at sites of trauma (secondary to intervention) in the coronaries may act as a triggering factor.

\section{CONFLICTS OF INTEREST |}

The authors declare that they have no conflicts of interest.

\section{REFERENCES}

1. Ahlehoff O, Gislason GH, Charlot M. Psoriasis is associated with clinically significant cardiovascular risk: A Danish nationwide cohort study. J Intern Med. 2011; 270: 147-157. doi: 10.1111/j.13652796.2010.02310.x
2. Ahlehoff $\mathrm{O}$, Lindhardsen J, Gislason GH, et al. Prognosis after Percutaneous coronary intervention in patients with psoriasis: A cohort Study using Danish nationwide registries. BMC Cardiovasc Disord. 2012; 12: 79-86. doi: 10.1186/1471-2261-12-79

3. Schwartz RS, Chronos NA, Varmani R. Preclinical restenosis models and drug eluting stents. Still important, still much to learn. J Am Coll Cardiol. 2004; 44(7): 1373-1385. doi: 10.1016/j. jacc.2004.04.060

4. Maksimowicz-McKinnon K, Selzer F, Manzi S, et al. Poor 1-year outcomes after percutaneous coronary interventions in systemic lupus erythematosus: Report from the National Heart, Lung, and Blood Institute Dynamic Registry. Circ Cardiovasc Interv. 2008; 1: 201-208. doi: 10.1161/CIRCINTERVENTIONS.108.788745

5. Desai SP, Januzzi JL, Pande AN, et al. Comparison of symptoms, treatment, and outcomes of coronary artery disease among rheumatoid arthritis and matched subjects undergoing percutaneous coronary intervention. Semin Arthritis Rheum. 2010; 40: 215-221. doi: 10.1016/j.semarthrit.2010.04.002

6. Thappa DM. The isomorphic phenomena of Koebner. Indian J Dermatol Venereol Leprol. 2004; 70: 187-189. 